\title{
Assessment of Lineages of Cercospora kikuchii in Louisiana for Aggressiveness and Screening Soybean Cultivars for Resistance to Cercospora Leaf Blight
}

\author{
Guohong Cai and Raymond W. Schneider, Department of Plant Pathology and Crop Physiology, Louisiana State \\ University Agricultural Center, Baton Rouge 70803; and Guy B. Padgett, Macon Ridge Research Station, Louisiana \\ State University Agricultural Center, Winnsboro 71295
}

\begin{abstract}
Cai, G., Schneider, R. W., and Padgett, G. B. 2009. Assessment of lineages of Cercospora kikuchii in Louisiana for aggressiveness and screening soybean cultivars for resistance to Cercospora leaf blight. Plant Dis. 93:868-874.

Cercospora leaf blight (CLB) became much more prevalent in Louisiana beginning in 1999. A previous study showed that the Louisiana population of Cercospora kikuchii, the causal agent, was dominated by a new lineage that differed from those collected in other locations at earlier times. In this study, we tested whether the dominance of the new lineage was caused by higher aggressiveness and screened soybean cultivars for resistance to CLB. Representative isolates from both lineages were used individually to inoculate six soybean cultivars in the greenhouse. Contrary to expectations, the new lineage was less aggressive. Three virulence groups were defined in this pathogen based on correlation of the aggressiveness of individual isolates on soybean cultivars. Other possible causes that led to the dominance of the new lineage in Louisiana are discussed. Eleven soybean cultivars were tested for disease reaction at two locations over 3 years in the field. Two cultivars, AG5701 (Asgrow) and TV59R85 (Terral), were among the more resistant cultivars to CLB both in the greenhouse and in the field.
\end{abstract}

Cercospora leaf blight (CLB) of soybean (Glycine max (L.) Merr.) is caused by Cercospora kikuchii (Tak. Matsumoto \& Tomoy.) M. W. Gardner (26). Under field conditions, symptoms of CLB begin to appear on upper leaves exposed to sunlight at the beginning of seed set. Leaves take on a light purple, leathery appearance, followed by the formation of reddish purple, angular-to-irregular lesions on upper and lower surfaces. Numerous infections cause rapid chlorosis and necrosis of leaf tissue, resulting in defoliation starting with the younger upper leaves. This fungus also infects soybean seed while still in the pod, where it causes purple seed stain $(11,24)$. Purple seed stain is characterized by purple, irregular blotches that range from small spots to complete coverage of the seed coat. Substantial yield losses caused by CLB have been reported $(12,22,28,29)$, and purple

Corresponding author: R. W. Schneider E-mail: rschnei@1su.edu

Current address of G. Cai: Department of Plant Biology and Pathology, Rutgers, The State University of New Jersey, New Brunswick 08901.

Accepted for publication 27 May 2009.

doi:10.1094/PDIS-93-9-0868

(C) 2009 The American Phytopathological Society seed stain affects seed quality and may cause reduced stands (12).

C. kikuchii produces cercosporin, a redpurple pigmented phytotoxin (17). Cercosporin absorbs light and transfers the energy to oxygen, producing singlet oxygen and superoxide ion, which are toxic to cells (13). Cercosporin plays a critical role in the ability of $C$. kikuchii to infect soybean plants. Mutants of an isolate of $C$. kikuchii that do not produce cercosporin failed to induce symptoms when inoculated onto soybean plants (25). This toxin is also an important pathogenicity factor in other diseases caused by cercosporinproducing Cercospora spp. $(7,8)$.

A number of soybean cultivars and genotypes have been screened for resistance to $C$. kikuchii. Different levels of resistance but not complete immunity have been reported. Plant introduction (PI) 80837 and cv. SJ2 are two of the genotypes more resistant to purple seed stain $(23,27)$, and resistance is likely controlled by a single dominant gene $(15,23)$. Recently, Xue et al. (30) reported that seed infection was positively correlated with oleic:linoleic acid ratio and oleic acid content in the soybean seed. Resistance to leaf infection, at least in some cultivars, is not related to resistance to seed infection. In greenhouse and field tests, some cultivars showed strong resistance to seed infection but were susceptible to CLB, while others had higher levels resistance to leaf infection but were susceptible to purple seed stain $(21,26)$.

C. kikuchii is an increasing threat to soybean production in many areas of the world $(28,29)$. In Louisiana, CLB became much more severe beginning in 1999 (19), and there has been a marked increase in the proportion of soybean cultivars that are susceptible to this disease in the field. In standardized cultivar trials conducted at that time, only a small proportion of the entries were susceptible, whereas, in 2002 , 59 of 62 cultivars were susceptible (22), and, in 2005, all 285 entries were susceptible (unpublished). Soybean growers in Louisiana have been shifting to earlymaturing cultivars in part to avoid CLB and other late-season diseases (18).

We have been using genetic and molecular markers to characterize the C. kikuchii population in Louisiana to determine possible causes of the rise of CLB. In the first study, vegetative compatibility grouping was used to characterize 58 isolates, of which 55 were collected in Louisiana during 2000 and 2001 (4). A high level of diversity with regard to vegetative compatibility groups (VCGs) was documented. In all, 56 self-compatible isolates were assigned to 46 VCGs, with only 6 VCGs containing multiple ( 2 or 3 ) isolates. High VCG diversity points to the possibility of a cryptic or recently lost sexual stage in this fungus. In a second study, random amplified polymorphic DNA and microsatelliteprimed polymerase chain reaction (PCR) were used to characterize 167 isolate of $C$. kikuchii (5). The Louisiana population was dominated by a new lineage (group I), and less than $5 \%$ of Louisiana isolates were grouped with those from outside sources (group II). Most isolates in the new lineage were divided into two subgroups (IA and IB). We also presented several lines of evidence that a cryptic sexual stage may be functioning in the new lineage. It is unclear whether the new lineage exists in other regions because only a limited number of isolates from other sources were included in the study, and they were collected much earlier. Other recent studies on C. kikuchii populations did not include isolates from the United States $(1,14)$. 
The dominance of group I and the rise of CLB in Louisiana point to the likelihood that this new lineage is responsible for the ascendancy of the disease. We hypothesized that (i) isolates in group I are more aggressive than those in group II or (ii) sexual recombination functions in group I and it gives this lineage a competitive advantage, such as in survival and dissemination. In this study, we tested whether the dominance of the new lineage was caused by higher aggressiveness and screened soybean cultivars for resistance to CLB.

\section{MATERIALS AND METHODS}

Greenhouse inoculations. Six soybean cultivars, AG5701 (Asgrow Seed Co., Stuttgard, AR), DP 5806RR (Delta and Pine Land Co., Scott, MS), HBK R5588 (Hornbeck Seed Co. Inc., DeWitt, AR), TV59R85 (Terral Seed Inc., Lake Providence, LA), SS RT 6299N (Southern States Coop., Richmond, VA), and DP 6880RR (Delta and Pine Land Co.), were included in the greenhouse tests. The first four cultivars are in maturity group (MG) $\mathrm{V}$, and the last two are in MG VI. These cultivars, as well as the cultivars included in field evaluations (see below), were chosen based on their overall performance (yield, value, and disease resistance) in previous years in a yearly standard field trial conducted by multiple researchers to evaluate soybean cultivars in Louisiana. Three soybean seeds of each cultivar were planted in a 1-liter plastic pot in a mixture of steam-disinfested soil:sand:Jiffy Mix (Jiffy Products of America, Inc., Batavia, IL), 1:1:2 (vol/vol/vol). Plants were fertilized with slow-release fertilizer (Osmocote, Marysville, $\mathrm{OH}$ ). After 10 days, plants were thinned to one plant per pot.

Nineteen isolates of $C$. kikuchii were randomly chosen to represent different lineages in the previous population study
(5). In all, 14 isolates were from group I (4 in group IA, 10 in group IB) and 5 were from group II (Table 1). Isolates were maintained on potato dextrose agar at $4^{\circ} \mathrm{C}$. Each isolate was transferred from the stock culture to a $10-\mathrm{cm}$-diameter plate containing $25 \mathrm{ml}$ of V8 agar (9) amended with $5 \mathrm{~g}$ of dried, senescent soybean leaf powder per liter (V8-SSLP) and incubated at $26^{\circ} \mathrm{C}$ under a 16 -h photoperiod for 6 days. Colonies were flooded with $10 \mathrm{ml}$ of sterile distilled water and rubbed with a glass rod. Half a milliliter of this conidial and mycelial suspension was used to inoculate each V8-SSLP plate. The plates were gently shaken to distribute the suspension across the agar surface. After incubation as described above, conidia in each plate were harvested in $20 \mathrm{ml}$ of sterile distilled water amended with $0.3 \%$ Tween 20 by gently rubbing the colonies with a glass rod. Suspensions were filtered through two layers of cheesecloth to remove mycelial clumps. The two-transfer procedure ensured high conidium yield. Conidial concentrations were determined with a hemacytometer and adjusted to $2 \times 10^{5} / \mathrm{ml}$.

Soybean plants were inoculated at the V3 growth stage (10). Conidial suspensions from each isolate were separately atomized onto soybean leaves from all directions until runoff. After inoculation, plants were placed in a moist chamber built with transparent plastic sheets on greenhouse benches. Plants inoculated with different isolates were placed approximately $0.7 \mathrm{~m}$ apart to minimize crosscontamination, and a plastic water tank was placed between groups to maintain high humidity. The moist chamber was closed at 6:00 p.m. and opened at 10:00 a.m. every day. After the chamber was closed, plants were sprayed with a fine mist for $30 \mathrm{~s}$. Daily average temperature in the chamber ranged from 20 to $28^{\circ} \mathrm{C}$ at night and 25 to $35^{\circ} \mathrm{C}$ during the daytime, and relative humidity ranged from 90 to $100 \%$ when the chamber was closed and 40 to $90 \%$ when the chamber was open. After 7 days, the plastic sheets were removed and plants were kept on the bench to allow disease development. Leaves above the second trifoliolate were removed every 3 days so that the inoculated leaves received direct sunlight. Disease severity was visually assessed on the two inoculated trifoliolate leaves with a standard area diagram (16) 21 days after inoculation. The experiment was conducted three times. Five plants from each cultivar were inoculated with each isolate in two tests, and four plants were used in the third test.

Leaf disk assays. When greenhousegrown soybean plants were at V3 growth stage, leaf disks (20 $\mathrm{mm}$ in diameter) were cut from two fully developed trifoliolate leaves with a sterile cork borer. Leaf disks were disinfested in a solution containing $46.5 \%$ ethanol and $2.6 \%$ sodium hypochlorite for $20 \mathrm{~s}$, washed in sterile distilled water three times, and air dried. Six leaf disks, one from each cultivar, were placed in a $10-\mathrm{cm}$-diameter petri dish containing $25 \mathrm{ml}$ of $1 \%$ water agar amended with benzimidazole at $50 \mathrm{ppm}$ (Sigma-Aldrich, St. Louis). Leaf disks were lightly pressed to the agar surface, and a $10-\mu l$ drop of conidial suspension $\left(2.0 \times 10^{5} / \mathrm{ml}\right)$ was placed in the center of each leaf disk. Disks in one plate were inoculated with the same isolate to avoid contamination. Sterile distilled water was used for controls, and five plates were used for each isolate and control. Plates were randomized and incubated under a 12-h photoperiod (light intensity $6,200 \mathrm{lux}$ ) at $27^{\circ} \mathrm{C}$ under light and $23^{\circ} \mathrm{C}$ in darkness. Eighteen days after inoculation, plates were photographed and the proportion of leaf disk area showing symptoms was measured with image analysis software (ASSESS; L. Lamari, Department of Plant Science, University of

Table 1. Pearson correlation coefficients between aggressiveness of individual isolates of Cercospora kikuchii on six soybean cultivars in greenhouse inoculations $^{\mathrm{z}}$

\begin{tabular}{|c|c|c|c|c|c|c|c|c|c|c|c|c|c|c|c|c|c|c|}
\hline \multirow[b]{2}{*}{ Isolate } & \multicolumn{18}{|c|}{ Isolate } \\
\hline & IA-1 & IA-2 & IA-3 & IA-4 & IB-1 & IB-2 & IB-3 & IB-4 & IB-5 & IB-6 & IB-7 & IB-8 & IB-9 & IB-10 & II-1 & II-2 & III-3 & II-4 \\
\hline IA-2 & 0.374 & & $\ldots$ & $\ldots$ & $\ldots$ & $\ldots$ & $\ldots$ & $\ldots$ & $\ldots$ & $\ldots$ & $\ldots$ & $\ldots$ & $\ldots$ & $\ldots$ & $\ldots$ & $\ldots$ & $\ldots$ & $\ldots$ \\
\hline IA-3 & 0.722 & 0.447 & $\ldots$ & $\ldots$ & $\ldots$ & $\ldots$ & $\ldots$ & $\ldots$ & $\ldots$ & $\ldots$ & $\ldots$ & $\ldots$ & $\ldots$ & $\ldots$ & $\ldots$ & $\ldots$ & $\ldots$ & $\ldots$ \\
\hline IA-4 & $\underline{0.920}$ & 0.497 & 0.675 & $\ldots$ & $\ldots$ & $\ldots$ & $\ldots$ & $\ldots$ & $\ldots$ & $\ldots$ & $\ldots$ & $\ldots$ & $\ldots$ & $\ldots$ & $\ldots$ & $\ldots$ & $\ldots$ & $\ldots$ \\
\hline IB-1 & $\overline{0.713}$ & 0.506 & 0.528 & $\underline{0.866}$ & . & $\ldots$ & $\ldots$ & $\ldots$ & $\ldots$ & $\ldots$ & $\ldots$ & $\ldots$ & $\ldots$ & $\ldots$ & $\ldots$ & $\ldots$ & $\ldots$ & $\ldots$ \\
\hline IB-2 & $\underline{0.989}$ & 0.370 & 0.725 & $\underline{0.936}$ & 0.795 & & $\ldots$ & $\ldots$ & $\ldots$ & $\ldots$ & $\ldots$ & $\ldots$ & $\cdots$ & $\ldots$ & $\ldots$ & $\ldots$ & $\ldots$ & $\ldots$ \\
\hline IB-3 & $\overline{0.782}$ & 0.037 & 0.279 & $\overline{0.767}$ & 0.780 & $\underline{0.831}$ & & $\ldots$ & $\ldots$ & $\ldots$ & $\ldots$ & $\ldots$ & $\ldots$ & $\ldots$ & $\ldots$ & $\ldots$ & $\ldots$ & $\ldots$ \\
\hline IB-4 & 0.594 & 0.780 & $\underline{0.879}$ & 0.608 & 0.448 & $\overline{0.571}$ & 0.047 & & $\ldots$ & $\ldots$ & $\ldots$ & $\ldots$ & $\ldots$ & $\ldots$ & $\ldots$ & $\ldots$ & $\ldots$ & $\ldots$ \\
\hline IB-5 & $\underline{0.912}$ & 0.498 & $\underline{0.934}$ & $\underline{0.828}$ & 0.629 & $\underline{0.900}$ & 0.513 & $\underline{0.836}$ & & $\ldots$ & $\ldots$ & $\ldots$ & $\ldots$ & $\ldots$ & $\ldots$ & $\ldots$ & $\ldots$ & $\ldots$ \\
\hline IB-6 & $\overline{0.678}$ & 0.345 & $\overline{0.563}$ & $\overline{0.859}$ & $\underline{0.961}$ & $\overline{0.763}$ & 0.753 & $\overline{0.404}$ & 0.610 & $\ldots$ & $\ldots$ & $\ldots$ & $\ldots$ & $\ldots$ & $\ldots$ & $\ldots$ & $\ldots$ & $\ldots$ \\
\hline IB-7 & $\underline{0.867}$ & 0.275 & 0.602 & 0.946 & 0.913 & $\underline{0.918}$ & $\underline{0.885}$ & 0.408 & 0.736 & $\underline{0.938}$ & & $\cdots$ & $\cdots$ & $\ldots$ & $\ldots$ & $\ldots$ & $\ldots$ & $\ldots$ \\
\hline IB-8 & 0.851 & 0.191 & 0.462 & $\overline{0.915}$ & 0.873 & $\overline{0.896}$ & 0.945 & 0.266 & 0.646 & 0.887 & 0.982 & $\ldots$ & $\ldots$ & $\ldots$ & $\ldots$ & $\ldots$ & $\ldots$ & $\ldots$ \\
\hline IB-9 & $\overline{0.908}$ & 0.212 & 0.655 & $\overline{0.892}$ & $\underline{0.861}$ & $\underline{0.956}$ & 0.906 & 0.409 & 0.795 & $\overline{0.864}$ & 0.966 & 0.947 & . & $\ldots$ & $\ldots$ & $\ldots$ & $\ldots$ & $\ldots$ \\
\hline IB-10 & $\underline{0.859}$ & 0.214 & 0.800 & $\overline{0.826}$ & $\overline{0.809}$ & $\overline{0.912}$ & $\overline{0.768}$ & 0.525 & $\underline{0.855}$ & $\overline{0.837}$ & $\overline{0.904}$ & $\overline{0.839}$ & $\underline{0.962}$ & $\ldots$ & $\ldots$ & $\ldots$ & $\ldots$ & $\ldots$ \\
\hline II-1 & $\overline{0.567}$ & 0.402 & 0.036 & $\overline{0.689}$ & 0.815 & $\overline{0.623}$ & 0.821 & 0.064 & $\overline{0.293}$ & $\overline{0.695}$ & $\overline{0.723}$ & $\overline{0.790}$ & $\overline{0.660}$ & 0.468 & & $\cdots$ & $\ldots$ & $\ldots$ \\
\hline II-2 & 0.623 & 0.436 & 0.188 & 0.546 & $\overline{0.173}$ & 0.523 & $\overline{0.333}$ & 0.375 & 0.464 & 0.067 & 0.317 & 0.378 & 0.291 & 0.137 & 0.399 & & $\ldots$ & $\ldots$ \\
\hline II-3 & 0.710 & 0.763 & 0.415 & 0.744 & 0.497 & 0.652 & 0.382 & 0.650 & 0.635 & 0.372 & 0.507 & 0.507 & 0.445 & 0.332 & 0.561 & 0.891 & $\ldots$ & $\ldots$ \\
\hline II-4 & 0.761 & 0.486 & 0.440 & 0.603 & 0.242 & 0.668 & 0.374 & 0.559 & 0.684 & 0.123 & 0.387 & 0.409 & 0.432 & 0.345 & 0.334 & $\underline{0.938}$ & $\underline{0.872}$ & $\ldots$ \\
\hline II-5 & 0.683 & 0.551 & 0.359 & 0.889 & 0.927 & 0.734 & 0.744 & 0.385 & 0.523 & 0.885 & 0.868 & 0.866 & 0.749 & 0.624 & 0.883 & $\overline{0.410}$ & $\overline{0.673}$ & 0.363 \\
\hline
\end{tabular}

${ }^{\mathrm{z}}$ Each isolate name starts with the lineage (IA, IB, or II) the isolate belongs to based on a previous study (5). Underlined values are significant at $P=0.05$. 
Manitoba, Winnipeg, Canada). The experiment was conducted twice.

Cercosporin toxicity assays. Cercosporin was extracted and purified according to a previously published procedure with modifications $(2,3)$. Mycelium of a 6week-old culture of $C$. kikuchii growing under light in potato dextrose broth was lyophilized and ground into a powder. Cercosporin was extracted with acetone and ethyl acetate. It was purified by passage through a dry hydroxyapatite column with an elution buffer of chloroform:methanol $(98.5: 1.5)$. Its purity was checked by thin-layer chromatography on silica gel G plates with a cercosporin standard (Sigma-Aldrich). The concentration of cercosporin was determined with a spectrophotometer (Spectronic 601; Milton Roy, Rochester, NY) using the molar extinction coefficient of $23,600 \mathrm{M}^{-1} \mathrm{~cm}^{-1}$ at an absorbance at $473 \mathrm{~nm}$ (31). Purified cercosporin was dissolved in acetone and stored at $-20^{\circ} \mathrm{C}$ in darkness.

Cercosporin toxicity was tested by plating leaf disks on water agar $(1 \%)$ containing $50 \mu \mathrm{M}$ cercosporin. Cercosporin in acetone stock was added to the medium after it was autoclaved and cooled to approximately $50^{\circ} \mathrm{C}$ with a final acetone concentration $3 \%$. Water agar containing $3 \%$ acetone was used as the control. Leaf disks (20 $\mathrm{mm}$ in diameter) were cut from two fully developed trifoliolate leaves from greenhouse-grown soybean plants at the V3 growth stage. Six leaf disks, one from each cultivar, were placed in each plate (10 $\mathrm{cm}$ in diameter) containing $25 \mathrm{ml}$ of medium. To facilitate the entry of cercosporin, leaf disks were pierced three times with a fine needle and lightly pressed to the agar surface. Plates were randomized and incubated under a 12-h photoperiod (light intensity $6,200 \mathrm{lux}$ ) at $27^{\circ} \mathrm{C}$ under light and $23^{\circ} \mathrm{C}$ in darkness. Nine days after inoculation, plates were photographed and the proportion of each leaf disk showing symptoms was measured as described above. The experiment was conducted twice.

Field experiments. Eleven soybean cultivars, including four used in greenhouse inoculations, were evaluated: AG5701, DP 5806RR, TV59R85, DK 5366RR (Delta
King Seed Co., McCrory, AK), HBK R5101 (Hornbeck Seed Co. Inc.), SS RT 5999N (Southern States Coop.), TV58R11 (Terral Seed Inc.), XR53N91 (Garst Seed Co., Slater, IA), SS RT 6202 (Southern States Coop.), DP 6880RR, and 96B21 (Pioneer Hi-Bred International, Inc.,
Johnston, IA). The first eight cultivars belong to MG V and the last three belong to MG VI. Soybeans were planted in midApril in 2002, 2003, and 2004 at research farms near Baton Rouge and Crowley, LA. Individual replications (plots) were four rows, $12.2 \mathrm{~m}$ in length on $0.8-\mathrm{m}$ centers,
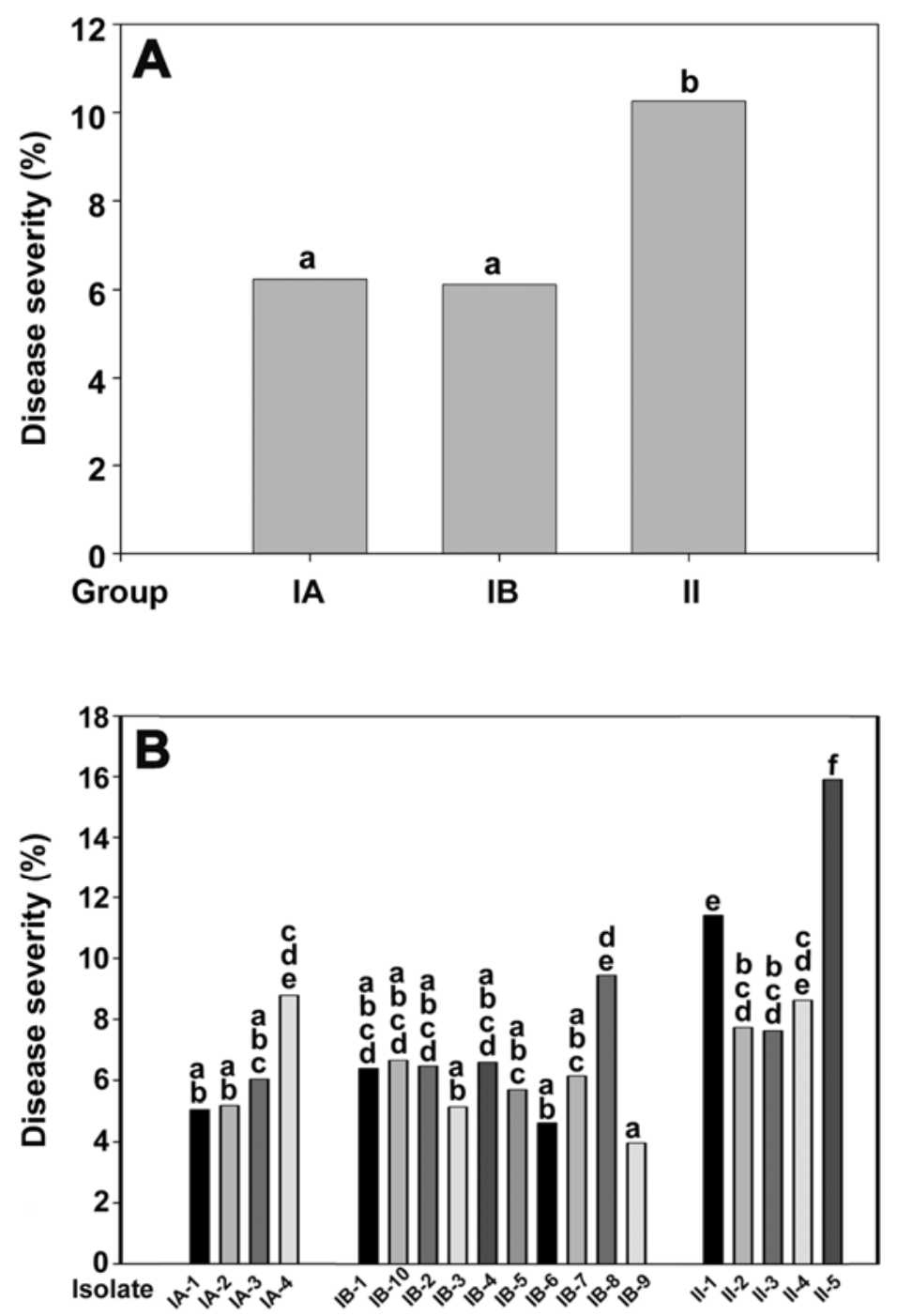

Fig. 2. Disease severity caused by $\mathbf{A}$, different lineages and $\mathbf{B}$, individual isolates of Cercospora kikuchii in greenhouse inoculations. Disease severity was measured as percentages of leaf area showing symptoms. Values (bars) whose labels (on top of the bars) do not share any letter were significantly different $(P=0.05)$. A, Groups I and II are different lineages in $C$. kikuchii identified in a previous population study, and IA and IB are the two subgroups in group I (5). B, Isolate designations start with the groups (IA, IB, and II) to which they belong.
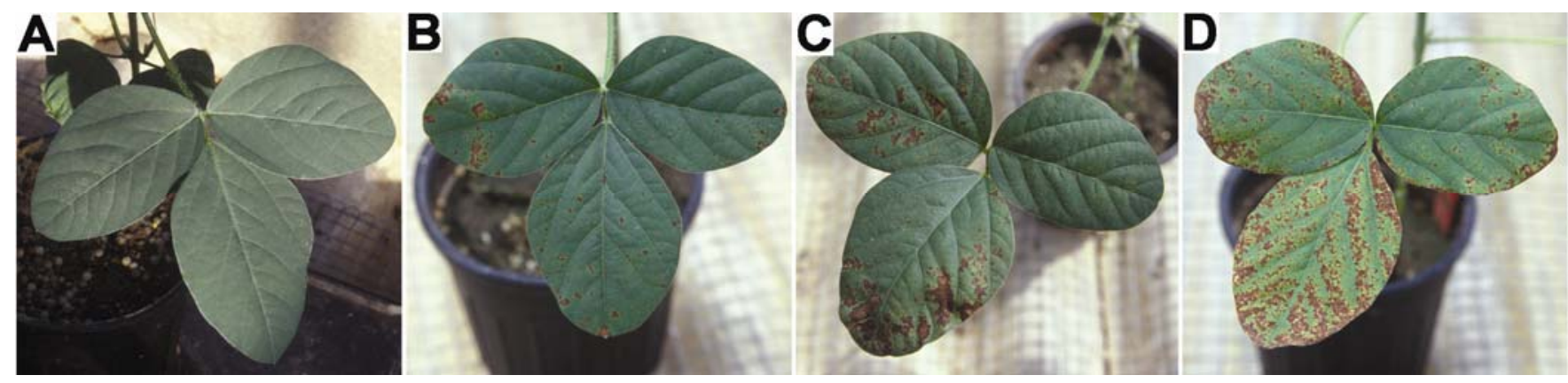

Fig. 1. Examples of leaf symptoms of Cercospora leaf blight of soybean in greenhouse inoculations. A, Control; B to D, infected leaves showing increasing disease severity. 
with a planting density of 23 seeds $/ \mathrm{m}$. There were four replications per cultivar at each location for each of the 3 years. Insects and weeds were managed according to recommended practices (20). There was no need to inoculate plants with spore suspensions because CLB had been widespread in Louisiana for several years. Disease severity was evaluated in the center two rows of each plot at the mid R6 stage of development (10) according to the following rating scale: 1 , no symptoms; 2 , $10 \%$ petioles with symptoms; 3 , as in 2 plus less than $10 \%$ affected leaves, including leaf veins; $4,10-25 \%$ of petioles and leaves with symptoms; $5,25-50 \%$ of petioles and leaves with symptoms; 6, 50$100 \%$ of petioles affected, but leaves do not have reddish cast and are not chlorotic; 7, upper leaves becoming reddened or chlorotic; 8, moderate chlorosis, reddening, and necrosis on most upper leaves; and 9 , as in 8 , but symptoms are severe plus defoliation.

Statistical analysis. The computer program Minitab (release 15; Minitab Inc., State College, PA) was used in statistical analyses. The general linear model was used in analysis of variance and in tests of interaction, and significance between means was tested using Tukey's pairwise comparison $(P=0.05)$.

\section{RESULTS}

Greenhouse inoculations. Examples of leaf symptoms are shown in Figure 1. Pinpoint, reddish purple spots appeared on inoculated leaves 6 to 7 days after inoculation. The spots later enlarged to angular-toirregular lesions, and several lesions coalesced to form necrotic areas. Chlorosis was observed in the surrounding areas. Only a few leaves developed a light purple, leathery appearance in the early stage of disease development. Veinal necrosis and lesions on petioles were observed in some plants. Higher disease severity was observed on the unifoliolate cotyledon leaves. They were not used for disease rating because the response of trifoliolate leaves (true leaves) was assumed to be more indicative of the response of the whole plant.

The new lineage (group I) was significantly less aggressive than the old lineage (group II) $(P<0.001)$. The two subgroups in group I were not significantly different $(P=0.96)$ (Fig. 2A). Detailed analyses of individual isolates showed that two of five isolates in the old lineage were significantly more aggressive than all or most other isolates (Fig. 2B).

Mean disease severity across all isolates ranged from $3.9 \%$ on AG5701 to $9.9 \%$ on DP 6880RR (Fig. 3A). Significant differences were detected among cultivars $(P<$ $0.001)$. Tukey's pairwise comparison tests divided six cultivars into three groups (Fig. 3A). The most resistant cultivars were AG5701 and TV59R85 and the least resis- tant cultivar was DP 6880RR. No cultivar was completely resistant to any isolate (Fig. 4B). The lowest disease severity was on cv. AG5701 with isolate IB-2 (1\%). Disease severity caused by individual isolates across cultivars did not always correlate. Pearson correlation coefficients $(r)$ of pairwise comparisons ranged from 0.036 to 0.989 and, of these, 53 were significant at $P=0.05$, indicating positive correlations (Table 1). Clustering analysis using correlation distance $(1-r)$ and single-linkage method identified three virulence groups, $\mathrm{a}, \mathrm{b}$, and c, with over $85 \%$ within-group
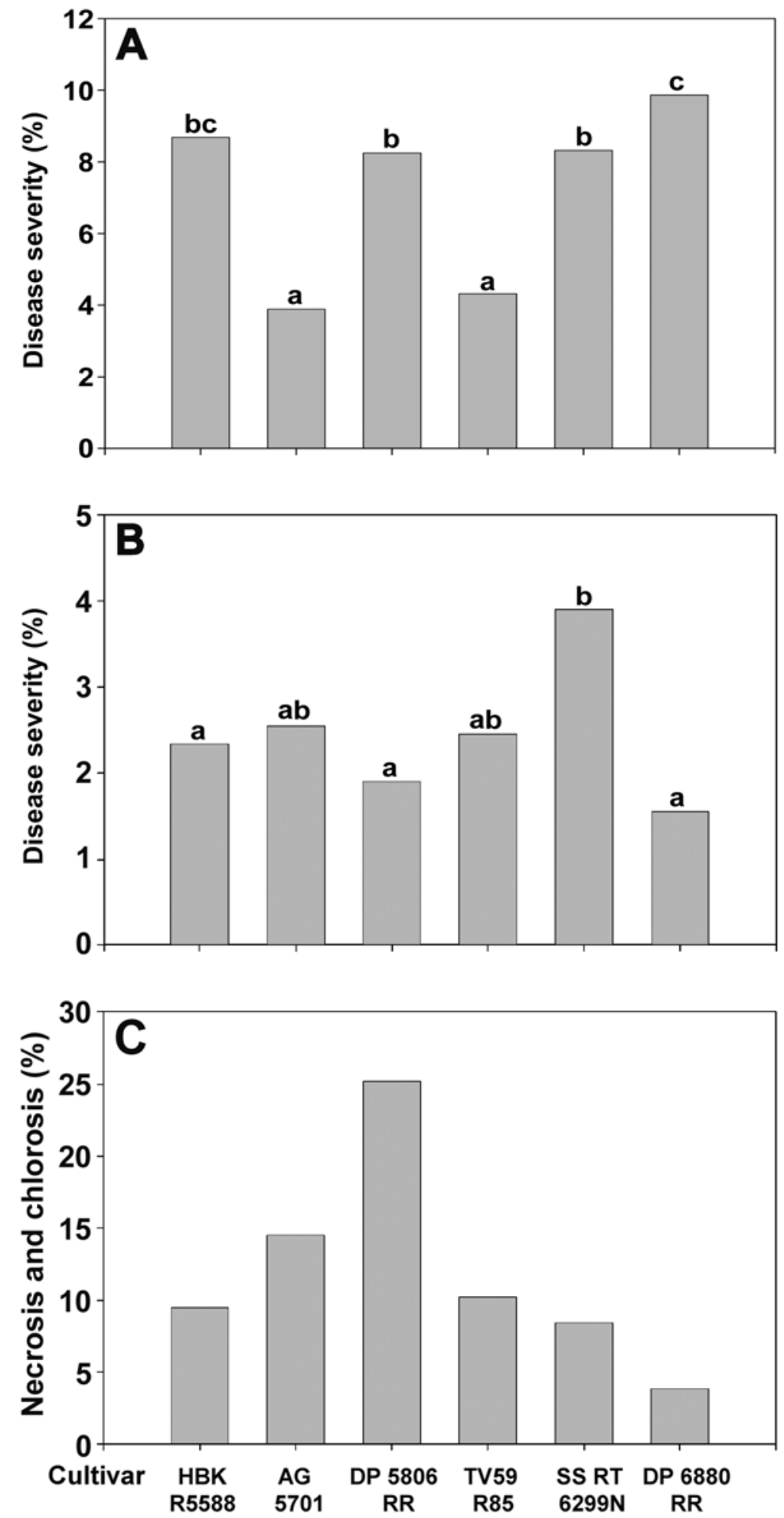

Fig. 3. Reactions of six soybean cultivars to Cercospora leaf blight in $\mathbf{A}$, greenhouse inoculations and $\mathbf{B}$, leaf disk assays and $\mathbf{C}$, to cercosporin. Disease severity was measured as percentages of leaf or leaf disk area showing symptoms. Toxicity of cercosporin was measured as percentages of leaf disk area showing necrosis and chlorosis. Values (bars) whose labels (on top of the bars) do not share any letter were significantly different $(P=0.05)$. None of the values in $\mathrm{C}$ was significantly different. 
similarity (Fig. 4A). There were significant correlations between isolates within but not between virulence groups. In group b, which included isolates from different lineages, isolates were positively correlated with only a subset of isolates in the same group (Table 1). The main difference between group a and the other two groups was that isolates in this group were similarly aggressive on cvs. HBK R5588 and AG5701, whereas those in the other two groups caused more disease in HBK R5588. The single isolate in group c differed from the other two groups in that it caused less disease on cv. SS RT 6299N than on cv. TV59R85, whereas the other two groups caused more disease in SS RT 6299N (Fig. 4B).

Leaf disk assays. It took approximately 10 days for symptoms to appear in most cultivars, and disease development was slow. Results from leaf disk assays did not correlate with greenhouse inoculations $(r=$ $-0.186, P_{>|\mathrm{r}|}=0.724$ ) (Fig. 3B). The most susceptible cultivar in greenhouse inoculations, DP 6880RR, had the least disease in the leaf disk assay. The two most resistant cultivars in greenhouse inoculations, AG5701 and TV59R85, were not significantly different from any other cultivars in these assays.

Cercosporin toxicity assays. Cercosporin induced necrosis at the wounds with
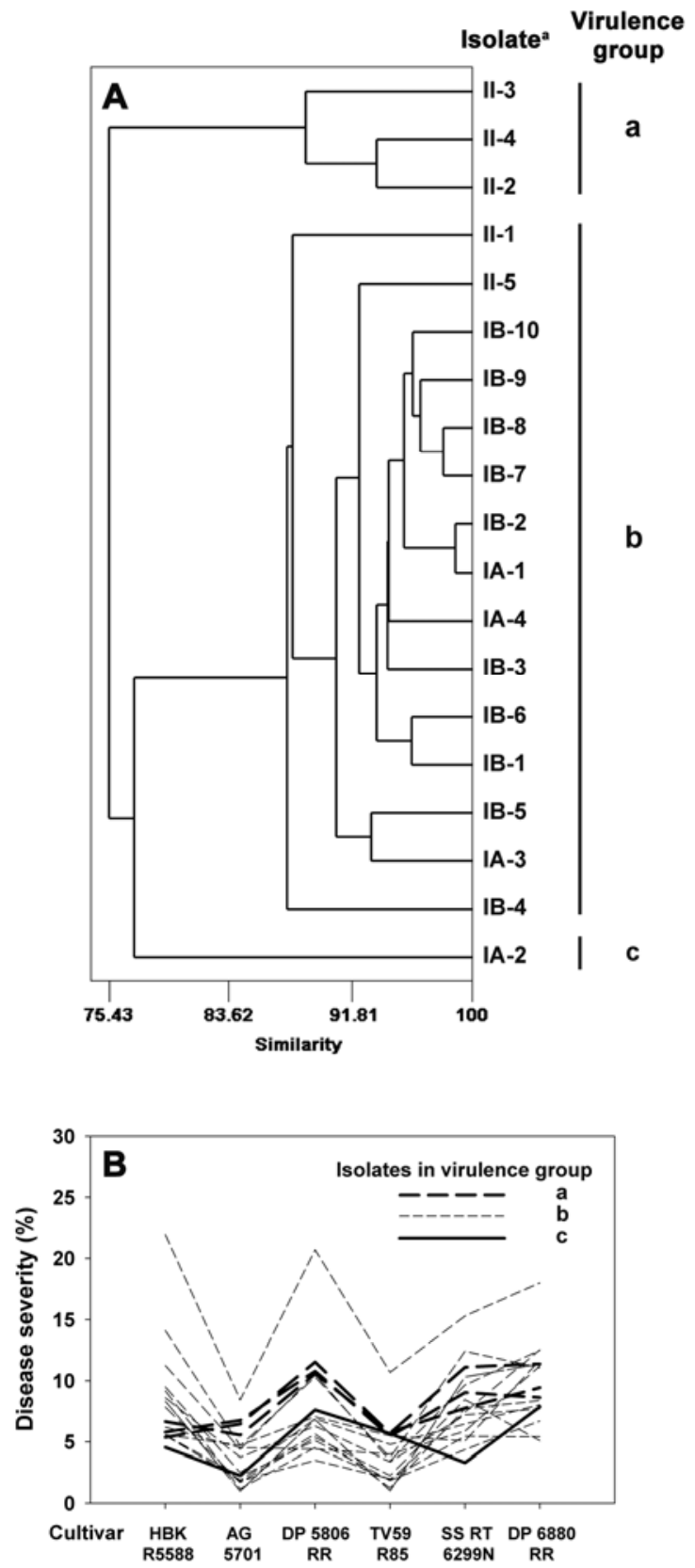

Fig. 4. Virulence groups in Cercospora kikuchii. A, Clustering analysis of the correlation of aggressiveness of individual isolates on soybean cultivars in greenhouse inoculations. Correlation distance ( 1 - Pearson correlation coefficient) and the single linkage method were used to construct the dendrogram. Isolate designations start with the groups (IA, IB, and II) to which they belong (5). B, Mean disease severity caused by individual isolates on each cultivar. This representation allows for a visual assessment of patterns by individual isolates across cultivars. chlorosis in the surrounding areas. A few leaves in the controls also developed small areas of chlorosis. The average percentages of leaf areas showing chlorosis in the controls of each cultivar were subtracted from those treated with cercosporin. Significant differences were not detected among cultivars $(P=0.472$; Fig. $3 C)$.

Field trials. There were significant interactions in disease severity in all combinations of cultivar, location, and year (Table 2). Among the 11 cultivars, DK 5366RR was most susceptible (Table 3). In agreement with the greenhouse inoculation results, cvs. AG5701 and TV59R85 were among the less susceptible cultivars and were significantly more resistant than $\mathrm{cv}$. DP 5806RR. Contrary to greenhouse results, in which cv. DP 6880RR was most susceptible, this cultivar was most resistant in the field. Disease severity continued to increase from 2002 to 2004 in both locations, and the disease was more severe in Crowley than in Baton Rouge in all 3 years (Table 3).

\section{DISCUSSION}

In our greenhouse inoculations, we routinely removed leaves above the inoculated leaves to ensure that the latter received abundant sunlight. Light is required for cercosporin toxicity and plays an important role in the development of diseases caused by cercosporin-producing Cercospora spp. $(8,25)$. In the field, CLB is first observed and most severe on upper leaves exposed to direct sunlight. We conducted two inoculations in the greenhouse without removing noninoculated leaves. Newly emerged leaves soon shadowed the inoculated leaves, and only sporadic purple spots were observed.

In a previous population study, we found that the C. kikuchii population in Louisiana was dominated by a new lineage (group I) (5). One possible explanation for the dominance of this new lineage is that it was more aggressive than the old lineage (group II). However, contrary to our expectations, it was less aggressive. The higher aggressiveness of group II was attributed to two isolates that were more aggressive than all or most isolates in group I, and the other three isolates in group II were not more aggressive than isolates in group I.

Table 2. Analysis of variance for Cercospora leaf blight severity on 11 soybean cultivars at two locations, Baton Rouge and Crowley, Louisiana and during 3 years (2002-2004)

\begin{tabular}{lrr}
\hline $\begin{array}{l}\text { Source of } \\
\text { variation }\end{array}$ & $\boldsymbol{F}$ & $\boldsymbol{P}>\boldsymbol{F}$ \\
\hline Cultivar (C) & 38.19 & $<0.001$ \\
Location (L) & 88.37 & $<0.001$ \\
Year (Y) & 35.52 & $<0.001$ \\
$\mathrm{C} \times \mathrm{L}$ & 17.17 & $<0.001$ \\
$\mathrm{C} \times \mathrm{Y}$ & 2.06 & 0.006 \\
$\mathrm{~L} \times \mathrm{Y}$ & 6.56 & 0.002 \\
$\mathrm{C} \times \mathrm{L} \times \mathrm{Y}$ & 3.43 & $<0.001$ \\
\hline
\end{tabular}


The alternative hypothesis for the dominance of group I in Louisiana is that cryptic sexual recombination may have been functioning in the new lineage, which gave it competitive advantages unrelated to aggressiveness. We previously described several lines of evidence that sexual recombination was functioning in the new lineage (4). Among these were the large number of VCGs in a relatively small collection, most isolates of which were in the new lineage; VCG was not an indicator of evolutionary lineage; a multilocus gametic disequilibrium test failed to reject the null hypothesis of random mating in group IB, a subgroup in the new lineage which included a majority of isolates in this lineage; and three haplotypes of the intergenic spacer region of ribosomal DNA were found in this organism, one of which appeared to be the recombination product of the other two (5). Assuming that sexual recombination occurs in the new lineage, this could result in fitness advantages related to survival, dissemination, or other traits. Also, infected soybean debris from past seasons may be a major source of inoculum, and sexual structures may be better adapted to overwintering or survival in the absence of the host. Of course, there are other possibilities that cannot be ruled out without direct experimentation. For example, isolates in the new lineage may produce more asexual spores or they may be more aggressive on soybean seed than those in the old lineage.

No isolate was avirulent on any cultivar, and differences in aggressiveness among individual isolates were quantitative rather than qualitative. Based on the correlation of their aggressiveness on soybean cultivars, isolates were assigned to three virulence groups. These groups differ from physiological races in that the latter are based on whether isolates are virulent or not on differential cultivars. The absolute aggressiveness of individual isolates within the same virulence group may differ greatly. For example, isolates IB-6 and II-5 differed more than threefold in absolute aggressiveness but their aggressiveness across tested soybean cultivars was positively correlated. That is, isolate II-5 was similarly more aggressive on each cultivar than isolate IB-6. Group B likely was a composite, in that isolates in this group were positively correlated with only a subset of isolates in the same group.

We did not inoculate the soybean plants in field trials. Because CLB had been endemic in Louisiana for several years, inoculation was unnecessary. In addition, results from inoculation with individual isolates in the field would likely be distorted by inoculum from the environment. The actual isolates that infected tested cultivars in the field probably were a mixture of multiple isolates that might belong to different lineages or virulence groups, and the composition may differ from year to year and location to location. Four cultivars were tested in the greenhouse and field, of which AG5701 and TV59R85 were consistently among the most resistant. They can serve as starting materials for breeding. Of course, their resistance to purple seed stain should be tested because resistance to leaf infection does not necessarily correlate with resistance to seed infection $(21,26)$. Cv. DP 6880RR was more susceptible than AG5701 and TV59R85 in greenhouse inoculations but not in field trials. In greenhouse inoculations, soybean cultivars other than DP 6880RR usually showed similar relative susceptibility to different $C$. kikuchii isolates, especially to those of the same virulence group, whereas relative susceptibility of DP 6880RR compared with other cultivars depended on the individual isolates used. One likely explanation for the discrepancy in DP 6880RR susceptibility between greenhouse inoculations and field trials is that CLB was caused by different isolates in the field.

From 2002 to 2004, CLB became more severe year after year in our field trials. This is in agreement with the overall trend of CLB in Louisiana during this period. The higher disease severity in Crowley than in Baton Rouge was probably caused by higher humidity in the former area. High humidity favors the development of CLB (12). The Crowley area had much higher rainfall. The large acreages of flooded rice in this area could also contribute to higher local humidity.

We intended to develop a detached leaf or leaf disk assay for CLB so that large numbers of cultivars could be readily screened. However, leaf disk assay results did not agree with greenhouse and field data. Also, development of this assay for CLB was hampered by the relatively long latent period required for symptom development. For greenhouse-grown plants, earliest symptoms appeared 5 days after inoculation, whereas up to 10 days were required in leaf disk assays. Although benzimidazole was used to retard senescence and the controls were green at the time they were scored, senescence was observed on mock-inoculated leaf disks shortly thereafter. Mycelial growth of the pathogen was abundant in the agar, and a small percentage of leaf disks had watersoaked areas when they were scored.

Batchvarova et al. (3) reported that a rice cultivar highly resistant to $C$. oryzae, which produces cercosporin, also was more resistant to the toxin than other lessresistant rice cultivars. In this study, soybean cultivars with different levels of resistance to CLB were not significantly different in their resistance to cercosporin. The data from cercosporin toxicity assays in this study had high coefficients of variation (56 to $414 \%$, depending on the cultivar). Although the highest mean was more than six times that of the lowest mean, no significant differences were detected. We also used other methods to test cercosporin toxicity on soybean plants. Leaves of greenhouse-grown plants were pierced with a needle, and a drop of cercosporin solution was added at the pierced sites. However, only a small percentage of

Table 3. Cercospora leaf blight disease severity ratings on 11 soybean cultivars grown at two locations during 3 years ${ }^{\mathrm{y}}$

\begin{tabular}{|c|c|c|c|c|c|c|c|}
\hline \multirow[b]{3}{*}{ Cultivar } & \multicolumn{6}{|c|}{ Year and location ${ }^{z}$} & \multirow[b]{3}{*}{ Average } \\
\hline & \multicolumn{2}{|c|}{2002} & \multicolumn{2}{|c|}{2003} & \multicolumn{2}{|c|}{2004} & \\
\hline & BR & CR & BR & CR & BR & CR & \\
\hline $96 \mathrm{~B} 21$ & 2.2 & 5 & 2.8 & 5.2 & 2.5 & 5.2 & $3.82 \mathrm{~d}$ \\
\hline AG5701 & 1 & 3 & 1.5 & 2 & 2.5 & 2.2 & $2.03 \mathrm{ab}$ \\
\hline DK 5366RR & 5 & 4.8 & 5.5 & 5.2 & 5.5 & 5.5 & $5.25 \mathrm{e}$ \\
\hline DP 5806RR & 2.8 & 2.8 & 2.2 & 3.8 & 2 & 4.2 & $2.97 \mathrm{c}$ \\
\hline DP 6880RR & 1 & 1 & 1 & 1.2 & 1.5 & 3.8 & $1.58 \mathrm{a}$ \\
\hline HBK R5101 & 1.5 & 1 & 2.8 & 1.8 & 3.8 & 1.5 & $2.07 \mathrm{ab}$ \\
\hline SS RT 5999N & 2 & 4.5 & 2 & 4.5 & 2.2 & 5.2 & $3.40 \mathrm{~cd}$ \\
\hline SS RT 6202 & 2.2 & 1.5 & 2.2 & 3.5 & 3.8 & 4.5 & $2.95 \mathrm{c}$ \\
\hline TV58R11 & 3.8 & 1.5 & 3 & 1.2 & 2.8 & 4.5 & $2.80 \mathrm{bc}$ \\
\hline TV59R85 & 1 & 1.5 & 1 & 3 & 2.8 & 3.5 & $2.13 \mathrm{ab}$ \\
\hline XR53N91 & 1.8 & 3.2 & 1.5 & 4.2 & 1.2 & 5.5 & $2.90 \mathrm{c}$ \\
\hline Average & $2.21 \mathrm{a}$ & $2.71 \mathrm{~b}$ & $2.32 \mathrm{ab}$ & $3.24 \mathrm{c}$ & $2.78 \mathrm{bc}$ & $4.15 \mathrm{~d}$ & $\ldots$ \\
\hline
\end{tabular}

${ }^{\mathrm{y}}$ Values that do not share the same letters within rows or columns are significantly different $(P=0.05)$. Rating scale: $1=$ no symptoms; $9=$ severe symptoms plus defoliation.

z BR and CR = Baton Rouge and Crowley, LA, respectively. 
treated leaves developed halos (data not shown). Injecting cercosporin directly into leaves was not successful because the leaves were too thin. Other methods, such as testing the effects of cercosporin on seedling growth, electrolyte leakage from leaf disks, and growth and survival of plant calli and cell suspensions $(3,6)$ may be useful. It remains to be determined whether resistance to CLB correlates with resistance to cercosporin.

\section{ACKNOWLEDGMENTS}

We thank the Louisiana Soybean and Grain Research and Promotion Board for financial support.

\section{LITERATURE CITED}

1. Almeida, A. M. R., Piuga, F. F., Marin, S. R. R., Binneck, E., Sartori, F., Costamilan, L. M., Teixeira, M. R. O., and Lopes, M. 2005. Pathogenicity, molecular characterization, and cercosporin content of Brazilian isolates of Cercospora kikuchii. Fitopatol. Bras. 30:594602.

2. Balis, C., and Payne, M. G. 1971. Triglycerides and cercosporin from Cercospora beticola: Fungal growth and cercosporin production. Phytopathology 61:1477-1484.

3. Batchvarova, R. B., Reddy, V. S., and Bennett, J. 1992. Cellular resistance in rice to cercosporin, a toxin of Cercospora. Phytopathology 82:642-646.

4. Cai, G., and Schneider, R. W. 2005. Vegetative compatibility groups in Cercospora kuchii, the causal agent of Cercospora leaf blight and purple seed stain in soybean. Phytopathology 95:257-261.

5. Cai, G., and Schneider, R. W. 2008. Population structure of Cercospora kikuchii, the causal agent of Cercospora leaf blight and purple seed stain in soybean. Phytopathology 98:823-829.

6. Daub, M. E. 1982. Peroxidation of tobacco membrane lipids by the photosensitizing toxin, cercosporin. Plant Physiol. 69:1361-1364.

7. Daub, M. E., and Ehrenshaft, M. 2000. The photoactivated Cercospora toxin cercosporin:
Contributions to plant disease and fundamental biology. Annu. Rev. Phytopathol. 38:461-490.

8. Daub, M. E., Herrero, S., and Chung, K.-R. 2005. Photoactivated perylenequinone toxins in fungal pathogenesis of plants. FEMS Microbiol. Lett. 252:197-206.

9. El-Gholl, N. E., Alfieri, S. A., Ridings, W. H., and Schoulties, C. L. 1982. Growth and sporulation in vitro of Cercospora apii, Cercospora arachidicola, Cercospora kikuchii, and other species of Cercospora. Can. J. Bot. 60:862868.

10. Fehr, W. R., Caviness, C. E., Burmood, D. T., and Pennington, J. S. 1971. Stage of development descriptions for soybeans, Glycine max (L.) Merrill. Crop Sci. 11:929-931.

11. Gardner, M. W. 1926. Indiana plant diseases, 1924. Proc. Indiana Acad. Sci. 35:237-257.

12. Hartman, G. L., Sinclair, J. B., and Rupe, J. C. (eds.) 1999. Compendium of Soybean Diseases. American Phytopathological Society Press, St. Paul, MN

13. Hartman, P. E., Dixon, W. J., Dahl, T. A., and Daub, M. E. 1988. Multiple modes of photodynamic action by cercosporin. Photochem. Photobiol. 47:699-703.

14. Imazaki, I., Homma, Y., Kato, M., Vallone, S., Yorinori, J. T., Henning, A. A., Iizumi, H., and Koizumi, S. 2006. Genetic relationships between Cercospora kikuchii populations from South America and Japan. Phytopathology 96:1000-1008

15. Jackson, E. W., Fenn, P., and Chen, P. Y. 2006. Inheritance of resistance to purple seed stain caused by Cercospora kikuchii in PI 80837 soybean. Crop Sci. 46:1462-1466.

16. James, W. C. 1971. An illustrated series of assessment keys for plant diseases, their preparation and usage. Can. Plant Dis. Surv. 51:3965.

17. Kuyama, S., and Tamura, T. 1957. Cercosporin. A pigment of Cercosporina kikuchii Matsumoto et Tomoyasu. I. Cultivation of fungus, isolation and purification of pigment. J. Am. Chem. Soc. 79:5725-5726.

18. Moore, S. H., and Boquet, D. J. 2008. Evaluating very early maturing soybeans in Louisiana. La. Agric. 51(1):28-29.
19. Moore, S. H., and Wolcott, M. C. 2000. Using yield maps to create management zones in field crops. La. Agric. 43(3):12-13.

20. Morrison, W. C., ed. 1996. Louisiana Soybean Handbook. La. Coop. Ext. Serv. Publ. 2624.

21. Orth, C. E., and Schuh, W. 1994. Resistance of 17 soybean cultivars to foliar, latent, and seed infection by Cercospora kikuchii. Plant Dis. 78:661-664.

22. Schneider, R. W., Bollich, P. K., and Harville, B. G. 2003. Evaluation of soybean cultivars for reactions to three foliar diseases. B\&C Tests (online) 18:P003 ( DOI:10.1094/BC18)

23. Srisombun, S., and Supapornhemin, P. 1993. Inheritance of soybean resistance to purple seed stain. Soybean Genet. Newsl. 20:92-93.

24. Suzuki, K. 1921. Studies on the cause of "Shihan" of soybean. Chosen Nakaiho 16:24-28.

25. Upchurch, R. G., Walker, D. C., Rollins, J. A., Ehrenshaft, M., and Daub, M. E. 1991. Mutants of Cercospora kikuchii altered in cercosporin synthesis and pathogenicity. Appl. Environ. Microbiol. 57:2940-2945.

26. Walters, H. J. 1980. Soybean leaf blight caused by Cercospora kikuchii. Plant Dis. 64:961-962.

27. Wilcox, J. R., Laviolette, F. A., and Martin, R. J. 1975. Heritability of purple seed stain resistance in soybeans. Crop Sci. 15:525-526.

28. Wrather, J. A., Anderson, T. R., Arsyad, D. M. Gai, J., Ploper, L. D., Porta-Puglia, A., Ram, H. A., and Yorinori, J. T. 1997. Soybean disease loss estimates for the top 10 soybean producing countries in 1994. Plant Dis. 81:107110 .

29. Wrather, J. A., Anderson, T. R., Arsyad, D. M. Tan, Y., Ploper, L. D., Porta-Puglia, A., Ram, H. H., and Yorinori, J. T. 2001. Soybean disease loss estimates for the top ten soybeanproducing countries in 1998. Can. J. Plant Pathol. 23:115-121.

30. Xue, H. Q., Upchurch, R. G., and Kwanyuen, P. 2008. Relationships between oleic and linoleic acid content and seed colonization by Cercospora kikuchii and Diaporthe phaseolorum. Plant Dis. 92:1038-1042.

31. Yamazaki, S., and Ogawa, T. 1972. Chemistry and stereochemistry of cercosporin. Agric Biol. Chem. 36:1707-1718. 